\title{
Towards Automating the Data Analytics Process
}

\author{
Chris Williams \\ Institute for Adaptive and Neural Computation \\ School of Informatics, University of Edinburgh \\ 10 Crichton Street, Edinburgh EH8 9AB, UK \\ ckiw@inf.ed.ac.uk
}

\begin{abstract}
The practical work of deploying a machine learning system is dominated by issues outside of training a model; this includes understanding the data set, data preparation and cleaning, model interpretation and explanation etc. The goal of the Artificial Intelligence for Data Analytics (AIDA) project at the Alan Turing Institute (UK) is to help to automate the whole data analytics process by drawing on advances in AI and machine learning. I will describe tools developed in AIDA to address such tasks, including identifying syntactic and semantic data types, data integration, and identifying and repairing missing and anomalous data. I will also discuss the prospects for further automation of the data analytics process.
\end{abstract}

